\title{
Cell transplantation preserves cardiac function after infarction by infarct stabilization: Augmentation by stem cell factor
}

\author{
Shafie Fazel, MD, MSc, Liwen Chen, MSc, Richard D. Weisel, MD, Denis Angoulvant, MD, MSc, \\ Charit Seneviratne, PhD, Amir Fazel, BSc, Phillip Cheung, BSc, Jason Lam, BSc, Paul W. M. Fedak, MD, PhD, \\ Terrence M. Yau, MD, MSc, and Ren-Ke Li, MD, PhD
}

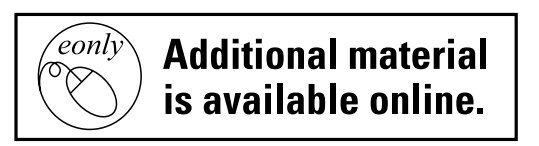

From the Division of Cardiac Surgery, Toronto General Hospital, University of Toronto, Toronto, Ontario, Canada.

This work was supported by operating grants from the Canadian Institute of Health Research (CIHR MOP14795) to R.K.L. and S.F., Heart and Stroke Foundation (HSF T5287) to R.D.W. and S.F., and the Physician Services Incorporated Foundation (R04-23) to S.F. and T.M.Y. S.F. is a CIHR and joint CIHR/HSF TACTICS Research Fellow and a Medical Scientist Training Fellow of the McLaughlin Centre for Molecular Medicine.

Read at the C. Walton Lillehei Resident Competition at the Eighty-fifth Annual Meeting of The American Association for Thoracic Surgery, San Francisco, Calif, April 10-13, 2005.

Received for publication April 7, 2005; revisions received June 30, 2005; accepted for publication July 19, 2005.

Address for reprints: Ren-Ke $\mathrm{Li}, \mathrm{MD}$, $\mathrm{PhD}$, NU 1-115, 200 Elizabeth Street, Toronto General Hospital, Toronto, Ontario, Canada M5G-2C4 (E-mail: renkeli@ uhnres.utoronto.ca).

J Thorac Cardiovasc Surg 2005;130:1310-8 $0022-5223 / \$ 30.00$

Copyright ( $\odot 2005$ by The American Association for Thoracic Surgery

doi:10.1016/j.jtcvs.2005.07.012
Objective: We hypothesized that implantation of adult mesenchymal stem cells after acute myocardial infarction mobilizes bone marrow precursor cells by activating the stem cell factor pathway, and that overdriving this pathway would enhance the beneficial effects of cell transplantation.

Methods: After coronary ligation, medium, mesenchymal stem cells, or stem cell factor-overproducing mesenchymal stem cells were injected into the anterior left ventricle. Cells from $\beta$-galactosidase transgenic mice enabled tracking of injected cells. The global and local impact of the cells was evaluated by measuring cytokine levels, endothelial progenitor cells, and myocardial angiogenesis, and by addressing cardiomyogenesis with confocal microscopy. The impact on cardiac function was evaluated by pressure-volume loops. Ventricular morphometrics were measured after in situ perfusion-fixation of the hearts at physiologic pressures.

Results: Implantation of mesenchymal stem cells increased myocardial stem cell factor levels 2.0-fold, endothelial progenitor cell mobilization 2.7-fold, and myocardial angiogenesis 2.3 -fold $(P<.05)$, but did not induce mitogenesis in host cardiomyocytes or give rise to $\beta$-galactosidase-expressing cardiomyocytes. Celltransplanted groups had improved indices of cardiac function, including preload recruitable stroke work and end-systolic elastance $(P<.001)$. Cell transplantation resulted in 2.0-fold smaller ventricular volumes $(P=.001)$ and 2.0 -fold reduced infarct scar area $(P=.056)$, but had no effect on the volume of spared myocardium. Stem cell factor overproduction imparted greater functional benefit without inducing detectable histologic cardiomyocyte regeneration.

Conclusion: Mesenchymal stem cell implantation after myocardial infarction facilitates functional cardiac regeneration without myocyte regeneration through augmentation of endogenous infarct repair, which is enhanced by stem cell factor.

$\mathrm{T}$ ransplantation of cultured bone marrow mesenchymal stem cells (MSCs) into the infarcted anterior left ventricle (LV) improved cardiac function in both rodent and porcine models. ${ }^{1,2}$ A subset of MSC, the multipotent adult progenitor cell, was recently identified that can differentiate to cells of all 3 germ layers, ${ }^{3}$ raising hopes that the MSC could form the basis of cell-based therapeutic strategies for cardiac regeneration. ${ }^{4}$ The mechanisms of action, however, remain unclear. The functional benefits of cell transplantation may derive from important paracrine effects of the cells on the local remodeling process as well as the recruitment and support of precursor cells important in tissue repair, but without the conversion of the cells to functioning cardiomyocytes.

Most precursor cells that have been shown to improve cardiac function after direct myocardial injection express the proto-oncogene c-kit tyrosine kinase recep- 


$$
\begin{aligned}
& \text { Abbreviations and Acronyms } \\
& \begin{array}{ll}
\mathrm{CD} & =\text { cluster of differentiation } \\
\mathrm{EPC} & =\text { endothelial progenitor cell } \\
\mathrm{LV} & =\text { left ventricle } \\
\mathrm{MI} & =\text { myocardial infarction } \\
\mathrm{MSC} & =\text { mesenchymal stem cell } \\
\mathrm{PECAM} & =\text { platelet endothelial cell adhesion molecule } \\
\mathrm{SCF} & =\text { stem cell factor }
\end{array}
\end{aligned}
$$

tor, which is the receptor for stem cell factor (SCF)., $\mathrm{SCF} / \mathrm{c}$-kit signaling is critical for the maintenance, function, and activation of all c-kit-expressing cells, ${ }^{7}$ which include hematopoietic stem cells, ${ }^{8}$ endothelial progenitor cells (EPCs), ${ }^{9}$ and the recently identified cardiac stem cells. ${ }^{10}$ Reports of differentiation of all these c-kit ${ }^{+}$cells to a cardiomyocyte phenotype have been published ${ }^{6,10,11}$ but remain controversial. ${ }^{9,12}$

In light of this, we hypothesized that (1) MSC transplantation does not result in significant cardiomyogenesis, (2) MSC transplantation improves cardiac function and angiogenesis by mobilizing bone marrow-derived precursor cells such as EPC, and (3) overexpression of SCF by MSC further improves the beneficial effects of MSC transplantation after myocardial infarction (MI).

\section{Methods}

\section{Mesenchymal Stem Cell Isolation and Stem Cell Factor Transfection}

Bone marrow was flushed from the femur and tibia of $1 \mathrm{C} 57 \mathrm{~B} 1 / 6$ (Charles River, Wilmington, Mass) and 1 Rosa26 ( $\beta$-galactosidase transgenic, Jackson Laboratories, Bar Harbor, Maine) mice to establish the MSC lines as described. ${ }^{1}$ After 10 passages the cells expressed cluster of differentiation (CD) 81 and stem cell antigen-1 (Sca-1), but not CD31, 34, 45, and c-kit. In vitro X-gal staining confirmed that $100 \%$ of the MSCs from Rosa26 mice expressed $\beta$-galactosidase.

Bluescript plasmid vector containing mouse SCF was provided by Professor T. Kunisada, Gifu University Graduate School of Medicine. ${ }^{13}$ The SCF insert was subcloned into pCDNA3 mammalian expression vector. The purified plasmid was transfected using Lipofectamine per the manufacturer's instructions (Invitrogen, Carlsbad, Calif). Transfected cells were transferred to Iscove's modified Dulbecco's medium with $200 \mu \mathrm{g} / \mathrm{mL}$ G418 the next day.

\section{Animal Procedures}

The Animal Care Committee of the University Health Network approved all experimental procedures. Animals were sedated, intubated, ventilated, and maintained with $2 \%$ isoflurane. The left coronary artery was ligated through a thoracotomy. This typically results in the infarction of $26.2 \% \pm 1.5 \%$ of the $\mathrm{LV}$ at 24 hours. For cell transplantation 300,000 cells were suspended in $15 \mu \mathrm{L}$ of serum-free medium and injected in 3 injections across the area subtended by the ligated coronary artery in a predetermined series of maneuvers that were consistent among the groups.

\section{Laser Scanning Confocal Microscopy}

Frozen optimum cutting temperature-embedded (Sakura-Finetek Inc, Torrance, Calif) blocks $(\mathrm{n}=3$ per time point) were cut into $10-\mu \mathrm{mol} / \mathrm{L}$ sections, fixed with $-20^{\circ} \mathrm{C}$ acetone, blocked with $5 \%$ normal serum, incubated with $1^{\circ}$ antibodies, and detected with conjugated $2^{\circ}$ antibodies. Slides were examined using an Olympus FluoView-1000 confocal microscope (Tokyo, Japan).

\section{Immunohistochemistry}

Sections ( $\mathrm{n}=3$ per time point) were treated with $3 \%$ hydrogen peroxide, blocked with normal serum, incubated with $1^{\circ}$ antiCD31 antibody followed by biotinylated $2^{\circ}$ antibody, labeled with streptavidin-horseradish peroxidase, and developed with NovaRED substrate (Vector Laboratories, Burlingame, Calif). For X-gal staining samples were fixed with $4 \%$ paraformaldehyde and stained with $1 \mathrm{mg} / \mathrm{mL}$ 5-bromo-4-chloro-3-indolyl-b-D-galactopyranoside for 24 hours at $37^{\circ} \mathrm{C}$. For measurement of angiogenesis, 5 random fields in both border zones of the infarct were chosen, and the number of large CD31-expressing structures was counted.

\section{Endothelial Progenitor Cell Assay}

Because of the confusion regarding the representative cell-surface phenotype of EPC, we used a functional in vitro colony formation assay to quantify EPC as described. ${ }^{14}$

\section{Protein Quantification}

Protein samples $(250 \mu \mathrm{g})(\mathrm{n}=3$ per time point per group) were subjected to reducing sodium dodecyl sulfate-polyacrylamide gel electrophoresis. Separated proteins were transblotted onto a nitrocellulose membrane, blocked, and incubated overnight with $1^{\circ}$ SCF antibody (1:1000). The membranes were washed and incubated with horseradish peroxidase-conjugated $2^{\circ}$ antibody (1:3000), and developed with a chemiluminescence kit.

\section{Cardiac Function and Morphometry}

For pressure-volume analysis ( $\mathrm{n}=10$ per group), $1 \mathrm{~mL}$ heparinized saline was injected intraperitoneally 30 to 45 minutes before the procedure to fluid-load animals. Under positive pressure ventilation, a micromanometer and conductance 1.4F (Millar Instruments, Inc, Houston, Tex) catheter was introduced into the LV through the right carotid artery. Pressure-volume loops were obtained during brief apnea and before and after inferior vena caval occlusion. After evaluation, hearts and lungs were obtained and weighed. Lungs were then dried and reweighed to estimate total lung water (wet weight - dry weight).

Twenty-eight days after MI ( $n=5$ per group), the hearts were arrested and fixed at physiologic pressures. Hearts were then cut into 1-mm sections and photographed for morphometry and stained for histology.

\section{Data Presentation and Statistics}

Data are presented as mean \pm standard error of the mean with the following abbreviations: $\mathrm{N}$, noninfarcted control; $\mathrm{M}$, infarcted and media-injected control; $\mathrm{C}$, infarcted and injected with MSCs; and 

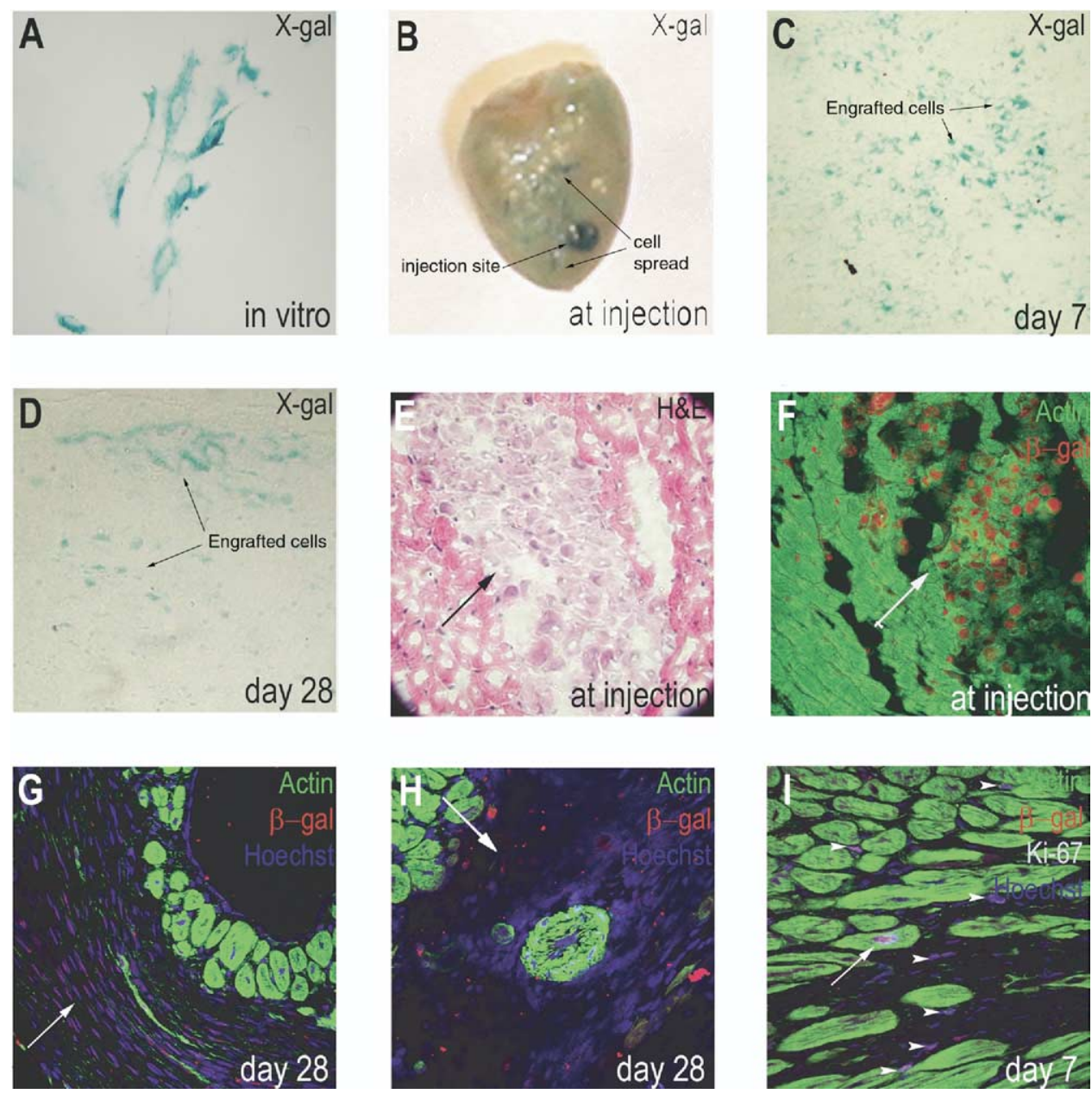

Figure 1. Tracking and the local impact of the implanted cells. A, X-gal staining of the MSC in vitro demonstrating that $100 \%$ of MSC prepared from $\beta$-galactosidase transgenic animals express $\beta$-galactosidase activity (blue) $(400 \times)$. B, X-gal staining of the heart immediately after 1 injection of the MSC showing the implanted cells as a raised blue area. $C, X$-gal staining of the infarcted section of the heart 7 days after cell implantation demonstrating significant engraftment of the cells $(200 \times)$. D, X-gal staining 28 days after implantation. Decreased number of surviving cells compared with day 7 is apparent $(200 \times)$. E, Hematoxylin-eosin staining demonstrating a cluster of injected cells (arrow) in mid-myocardium immediately after injection $(400 \times)$. F, Serial section of the same region as in (E) with actin labeling in green and $\beta$-galactosidase labeling in red. The implanted cells (arrow) are identifiable in the myocardium by red immunostaining. $G$, Similar labeling as above with nuclei in blue. Highly engrafted area 28 days after cell injection (arrow). $\beta$-Galactosidase-expressing cells (red) are visualized arranged in a circumferential orientation. The adjacent spared subendocardial myocytes do not express $\beta$-galactosidase. H, Similar labeling as in (F) demonstrating that despite engraftment (arrow) the MSCs have not contributed to the musculature or endothelial lining of the visualized blood vessel. I, Similar labeling as above with the addition of Ki67 staining in grey (arrowheads). Cardiomyocyte (arrow) in the cell cycle detected by the Ki67 antigen. The number of such cells was less than $0.1 \%$ in all groups.

CS, infarcted and injected with MSCs overexpressing SCF. Statistical analysis between 2 groups was performed using a 2-tailed $t$ test. Multiple comparisons were performed using analysis of variance with Bonferroni's posttest if the overall analysis was significant. Differences in preload recruitable stroke work and end-systolic elastance were tested using linear regression. Survival data were analyzed by Kaplan-Meier curves.

\section{Results}

Mesenchymal Stem Cell Implantation Does Not Induce Cardiomyocyte Regeneration

After coronary ligation, MSC (Figure 1, A) or medium was injected into the infarct zone. Implanted cells were identified by 2 techniques: X-gal enzymatic staining (Figure 1, 
A

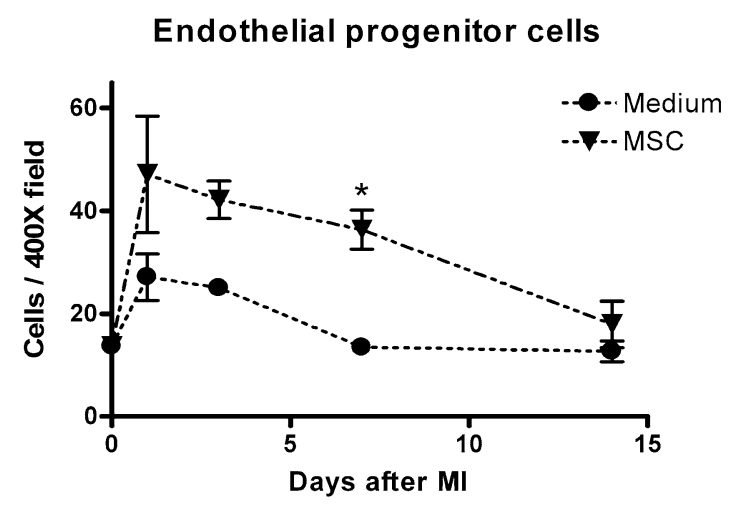

B

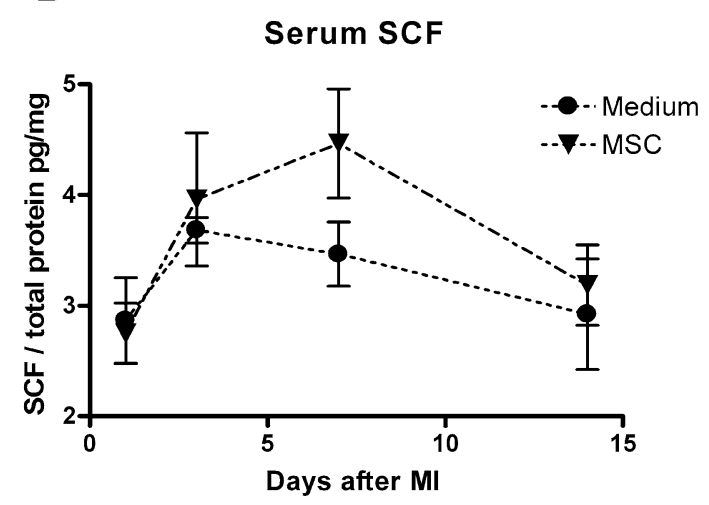

C

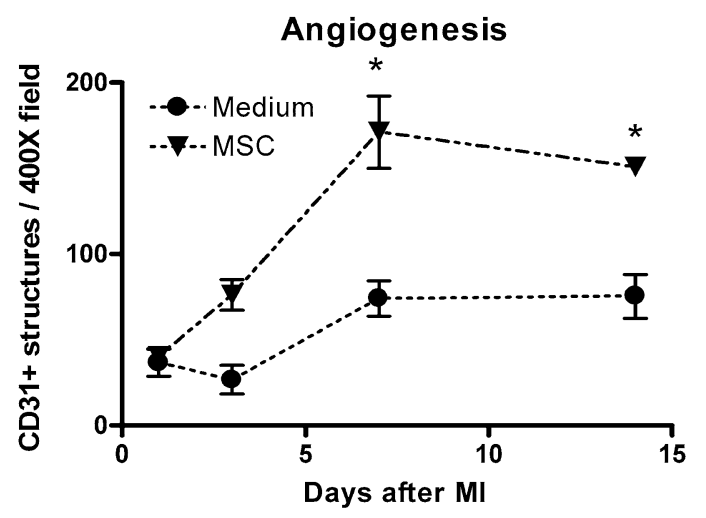

D

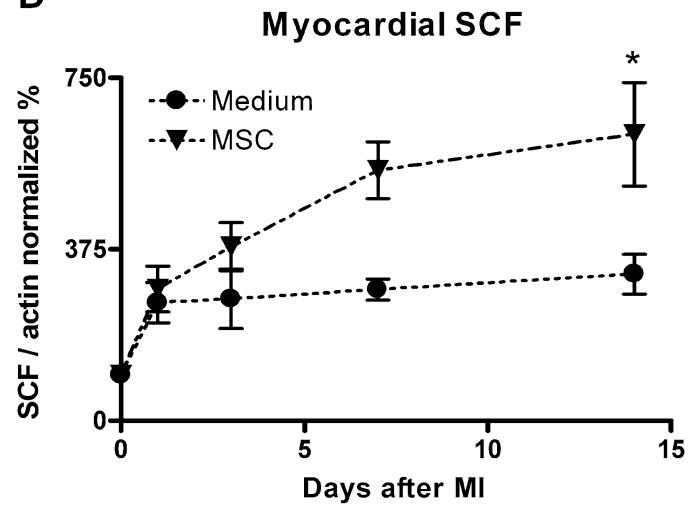

Figure 2. Cell implantation and angiogenesis (A-D). Graphs quantifying post-MI EPC mobilization (A), serum SCF (B), myocardial angiogenesis in the border zone (C), and myocardial SCF (D) ( $n=3$ per time point per group). ${ }^{*} \boldsymbol{P}<$.05. MSC, Mesenchymal stem cell; MI, myocardial infarction; $S C F$, stem cell factor.

$B-D)$ and light microscopy, and immunostaining and confocal microscopy (Figure 1, F-I). The cells were present in clusters in mid-myocardium after implantation (Figure 1, $C)$. Engraftment of $\beta$-galactosidase-expressing cells was observed to day 28 (Figure 2,D), the last time point at which this was evaluated. Qualitatively, the number of surviving cells decreased from day 7 to day 28 , as the cells lined circumferentially through the myocardial scar (Figure $1, C$ and $D$ ). However, neither immunostaining nor $\mathrm{X}$-gal staining identified $\beta$-galactosidase-expressing cardiomyocytes near engrafted areas in 30 border zones examined at various time points (Figure 1, $G$ and $H$ ). An estimated 13,350 border zone cardiomyocytes were scanned for this analysis. Similarly, MSC implantation did not increase cellcycle activity in adjacent cardiomyocytes as evaluated by expression of the Ki67 cycling antigen (Figure 1, I). In the 30 border zones at various time points that were subjected to this analysis, the observed rate of cell-cycle activity was less than $0.1 \%$, which was comparable to controls (Figure 1, I).

\section{Mesenchymal Stem Cell Implantation Mobilizes Endothelial Progenitor Cells and Improves Angiogenesis}

MI increased the number of peripheral EPCs that returned to baseline by day 7 in the medium group (Figure 2, A). The kinetic of EPC mobilization was enhanced in the MSC group $(P=.004)$ with a more gradual return to baseline. Concurrently, systemic SCF levels achieved a plateau on day 3 in the medium group, whereas in the MSC group, SCF continued to increase to day 7 peak values, 1.3 -fold higher than in the medium group $(P=.16$ ) (Figure 2, $B)$.

Angiogenesis was increased in the MSC group. The number of small capillary-like structures rapidly decreased after coronary ligation, but larger disorganized vessels became apparent starting on day 7 . The number of such vessels increased 2.3-fold in the MSC group when compared with controls $(P=.01)$ on day 7 (Figure 2, $C$ ). In the infarcted myocardium membrane isoform of SCF protein was increased 2.0-fold in the MSC group $(P=.04)$ com- 
A

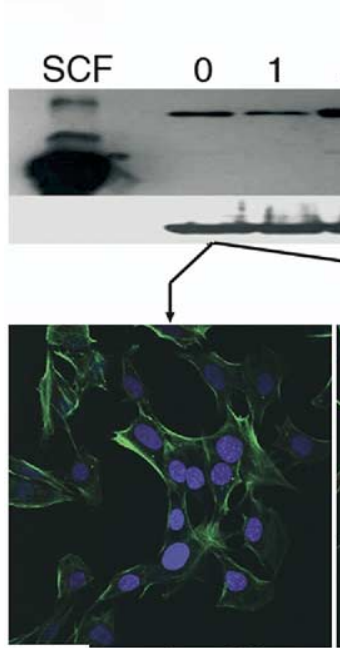

Parental cell line Staining control

\section{G418 G418}

$\begin{array}{llll}7 & 14 & P 0 & P 5\end{array}$

\section{SCF}
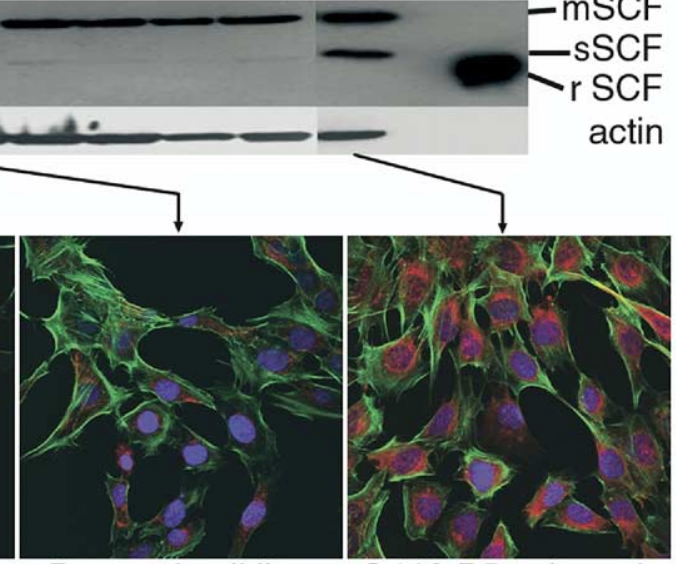

Parental cell line Stained for SCF

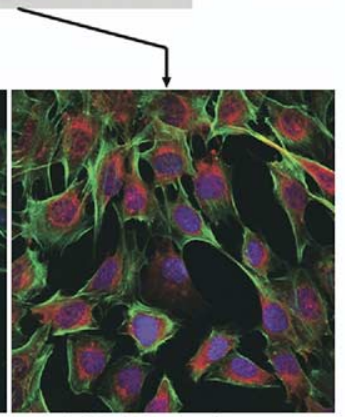

G418 P5 selected cell line

Stained for SCF
B

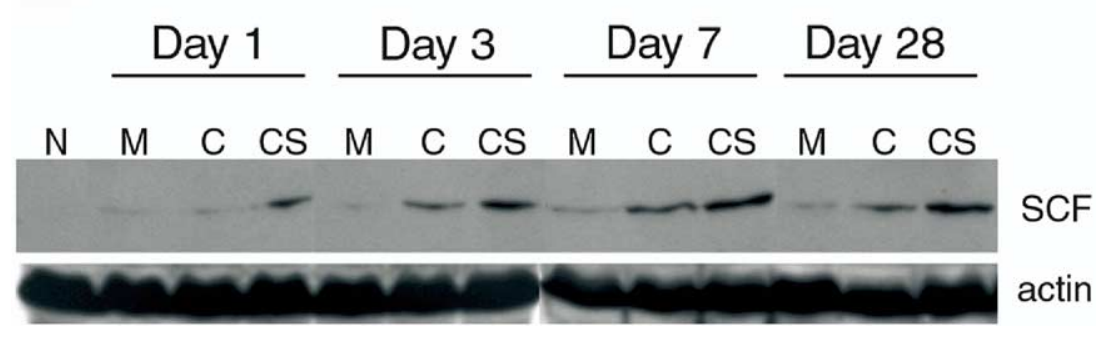

Figure 3. SCF enhancement of MSC. A, Immunoblot showing that MSCs express SCF in vitro (lane 0). Transient transfection up-regulates SCF production (lanes 1, 3, 7, and 14 denote days after transfection), and $\mathbf{G 4 1 8}$ selection (lanes PO and $P 5, P$ denotes passage number) yields a cell population with high-level expression of both membrane ( $m S C F$ ) and soluble (sSCF) isoforms. Recombinant SCF (rSCF) is the positive control. Confocal images of MSC (left and center lower panels) and the G418-selected population (lower right panel) probed for SCF by immunostaining. Staining control (left pane) showing no staining in the absence of the primary SCF antibody. The images demonstrate low-level SCF production under basal conditions, which is augmented with transfection and G418 selection (right pane). B, Immunoblot of infarcted segments over a time-course. MI upregulates infarct SCF levels. MSC implantation augments this endogenous response, which is further enhanced by the implantation of the stably transfected MSC-SCF clone. Immunoblot is representative of 3 independent experiments. SCF, Stem cell factor, $P$, passage number; $N$, non-infarcted control group; $M$, mediuminjected group; C, MSC-injected group; CS, MSC-SCF group. pared with medium-injected controls, which also had elevated SCF levels (Figure 2, D). The soluble isoform of SCF was not observed.

Stem Cell Factor Enhancement of Mesenchymal Stem Cells Improves Myocardial Repair After Infarction In vitro MSC expressed SCF at low levels, and transfection of SCF plasmid increased both membrane and soluble isoforms of SCF (Figure 3, A). We obtained a stably transfected heterogenous cell population by selection in G418 containing medium. This cell population expressed high levels of both isoforms of SCF in vitro (Figure 3,A). The doubling time of the G418-selected population and the nontransfected MSC was similar, and no morphologic changes in cell size and shape were observed.

After induction of MI, medium, MSC, or MSC-SCF was implanted into the infarcted area. The earliest and highest membrane SCF expression was observed in the MSC-SCF group (CS), followed by the MSC (C) and medium (M) groups (Figure 3, B). The MSC-SCF group had a 3.4-fold higher day 3 and 3.2-fold higher day 7 myocardial SCF than the MSC alone group $(P<.05)$. SCF was not upregulated in the noninjured region of the heart.

By 28 days after MI, 76.5\% of animals in the medium group, $90.6 \%$ of animals in the MSC group, and $86.3 \%$ of animals in the MSC-SCF group were alive (log-rank $P=$ .11). The surviving mice underwent evaluation of diastolic (Figure 4, A-D) and systolic cardiac function (Figure 4, $E-H)$. After fluid loading, end-diastolic pressures reached $26.9 \pm 0.9 \mathrm{~mm} \mathrm{Hg}$ and did not differ between groups (Figure $4, A$ ). Changes in $\mathrm{dP} / \mathrm{dt}$ min did not reach statistical significance, but cell implantation improved tau (Figure 4, $B$ and $C$ ). SCF enhancement improved tau and flattened the end-diastolic pressure volume relationship toward the noninfarcted controls (Figure 4,D). End-systolic pressures were on average $9.7 \mathrm{~mm} \mathrm{Hg}$ higher in the cell-transplanted groups compared with the medium group $(P=.04)$ (Figure $4, E$ ). Measurement of preload recruitable stroke work and end-systolic elastance demonstrated best cardiac perfor- 
A

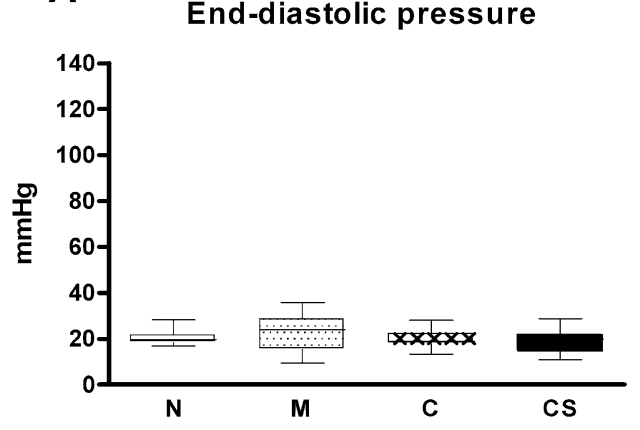

B

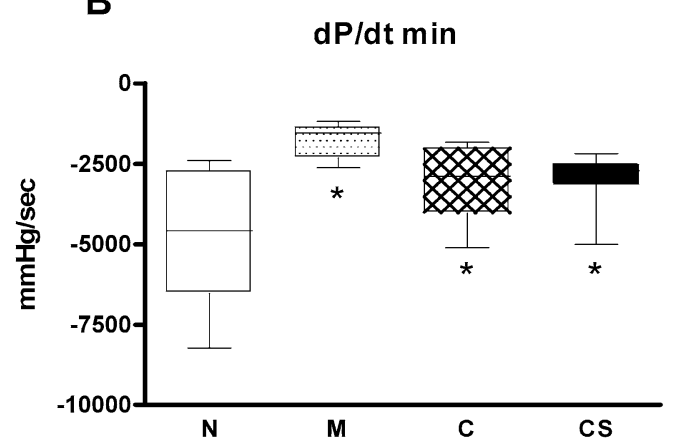

C
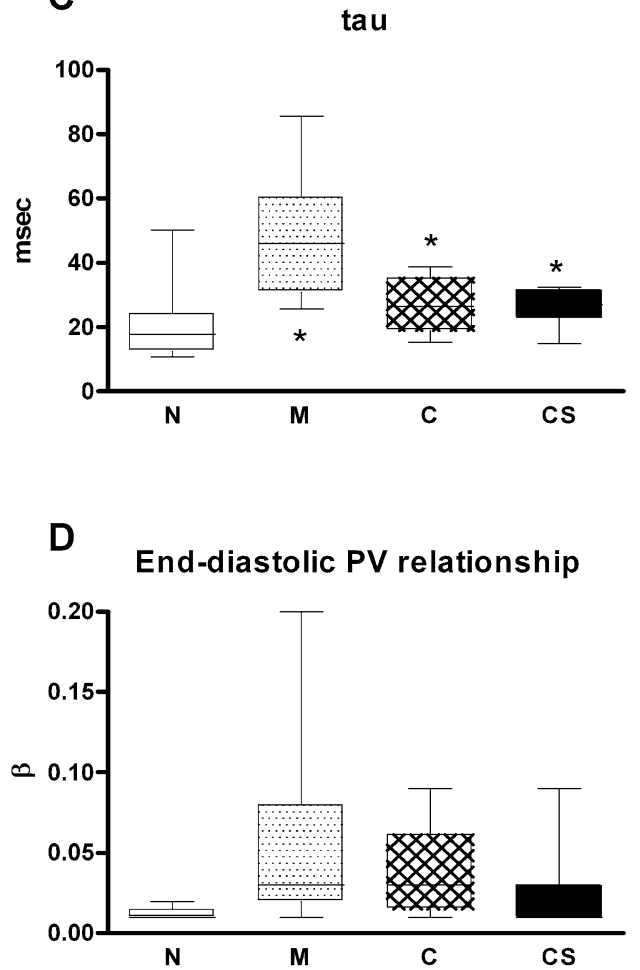

E

End-systolic pressure

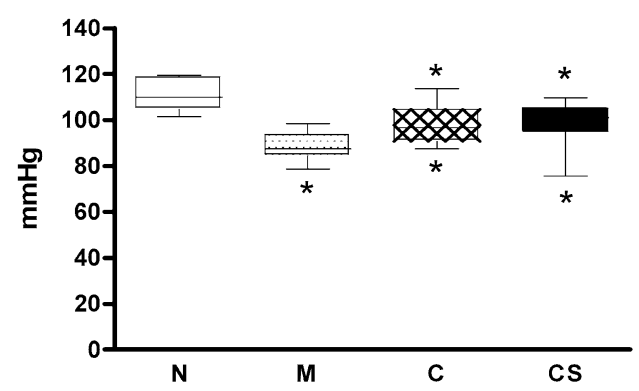

$\mathbf{F}$

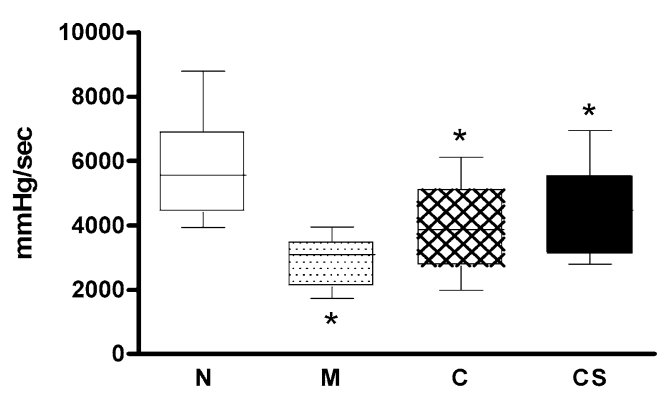

G

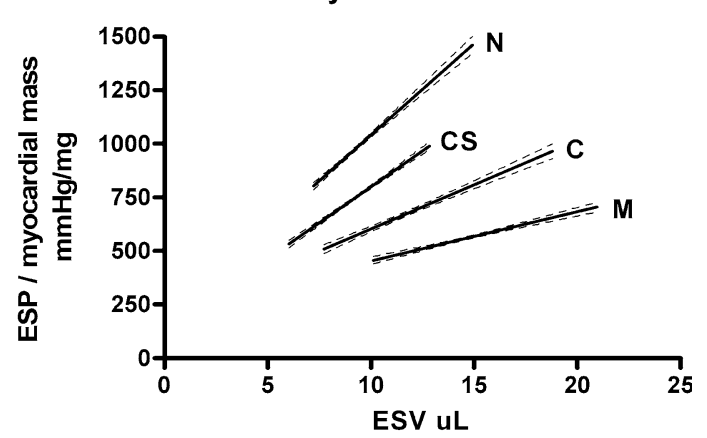

H

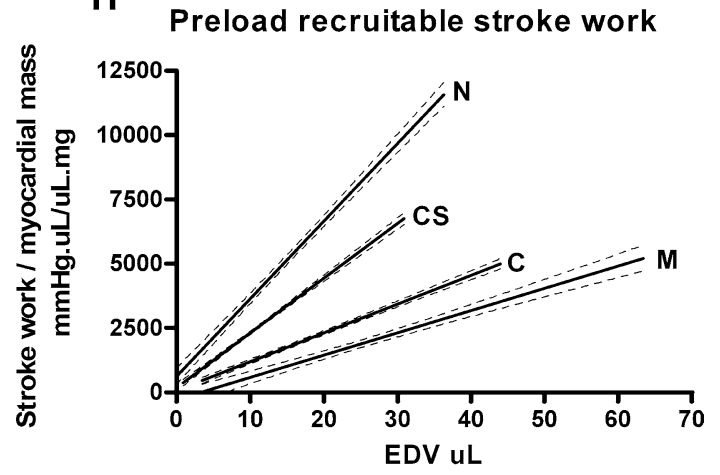

Figure 4. Summary of pressure-volume analysis. Assessment of diastolic (A-D) and systolic (E-H) function ( $n=10$ per group). N, noninfarcted control group; M, medium-injected group; C, MSC-injected group, CS, MSC-SCF group; asterisk ${ }^{*}$ ) below the box plots denotes $P<.05$ comparing treated groups with group $\mathrm{N}$; asterisk (*) above the box plots denotes $P<.05$ comparing $C$ and $C S$ groups with $M$. Differences in end-systolic elastance and preload recruitable stroke work between $\mathbf{N}$ and $\mathrm{M}, \mathrm{M}$ and $\mathrm{C}$, and $\mathrm{C}$ and $\mathrm{CS}$ were highly significant at $\boldsymbol{P}<.001$ using linear regression analysis. $P V$, Pressure-volume; ESV, end-systolic volume; EDV, end-diastolic volume; ESP, end-systolic pressure. 
mance in the noninfarcted group, followed by MSC-SCF, MSC, and finally the medium-injected groups $(P<.001)$ (Figure 4, $G$ and $H$ ).

Commensurate with these observations in the survivors, heart-to-body weight ratio, indicative of cardiac hypertrophy, was highest in the medium and MSC groups $(\mathrm{N}, 0.4 \pm$ $0.02 ; \mathrm{M}, 0.6 \pm 0.13 ; \mathrm{C}, 0.6 \pm 0.03 \mathrm{mg} / \mathrm{g} \cdot 100 ; P<.05, \mathrm{~N}$ vs $\mathrm{M}$, and $\mathrm{N}$ vs $\mathrm{C} ; P=$ not significant, $\mathrm{M}$ vs $\mathrm{C}$ ) and 0.8 -fold lower in the MSC-SCF group (CS, $0.5 \pm 0.01 ; P=.01, \mathrm{CS}$ vs M). Pulmonary congestion, as assessed by wet-to-dry lung weights, was not different between treatment groups. Morphometry of explanted hearts demonstrated that ventricular volumes were 2.0-fold larger in the medium group $(\mathrm{M}, 45.1 \pm 6.0 \mu \mathrm{L})$ compared with the cell-transplanted groups $(\mathrm{C}, 27.9 \pm 3.2$; CS, $19.8 \pm 1.6 \mu \mathrm{L} ; P<.05$, $\mathrm{M}$ vs $\mathrm{C}$, and $\mathrm{M}$ vs CS). Volume of spared myocardium was not different among the treatment groups. The surface area of the scar was largest in the medium-injected group (M, $8.2 \pm$ $0.9 \mathrm{~mm}^{2}$ ), followed by the MSC-injected group (C, $5.7 \pm$ $1.2 \mathrm{~mm}^{2}$ ). The scar area was significantly smaller in the MSC-SCF group (CS, $2.8 \pm 0.5 \mathrm{~mm}^{2}, P<.001$, CS vs M).

Histology of the infarcted region did not show ingrowth of myocytes in any of the groups. A thin layer of spared myocytes remained in the subendocardium (Figure E1).

\section{Discussion}

We made several observations in the present study: (1) MSC implantation after MI improved cardiac function and geometry without inducing cardiomyogenesis, (2) MSC implantation enhanced EPC mobilization and induced angiogenesis, (3) SCF, typically a cytokine associated with the maintenance of the bone marrow c-kit ${ }^{+}$stem and progenitor cells, is markedly upregulated in infarcted hearts and further increased after MSC implantation, and (3) increasing dysregulated expression of SCF by MSC augmented myocardial repair.

With the use of genetic tagging we were able to assess the fate of the injected cells. In our scanning of approximately 13,350 cardiomyocytes in the border zone of engrafted areas, we did not detect a single cardiomyocyte that bore the genetic tag of the injected cells as suggested by others. ${ }^{15}$ This was assessed by both immunostaining and confocal microscopy as well as by X-gal staining. The dual strategy was used to minimize the possibility of falsepositive results associated with immunofluorescent techniques. Similarly, adjacent to the implanted MSC, the observed rate of cardiomyocyte cell-cycle entry was less than $0.1 \%$, ruling out the possibility that implanted cells fuse with cardiomyocytes and induce them to enter the cell cycle as suggested by previous studies. ${ }^{16,17}$ Furthermore, regenerating bands of myocardium that could have derived from recruited stem cells were not observed by histology in the infarct core even when the cells overexpressed the stem cell survival and activation factor, SCF.

Even in the absence of induced cardiomyogenesis, MSC implantation prevented ventricular dilation and improved load-independent indices of cardiac function. The effects of MSC implantation included significantly improved angiogenesis by systemic effects on EPC mobilization, and improved local remodeling of the evolving infarct by preventing ventricular dilation. We believe that some of these effects were partially mediated through increased levels of SCF because regional overexpression of SCF by the cells further reduced infarct surface area and improved cardiac function. Although the MSCs are known to secrete $\mathrm{SCF},{ }^{18}$ the finding that MSCs secrete SCF and other cytokines after transplantation into a fresh MI has not been demonstrated. The current work raises the possibility that the expression of such cytokines by the MSC may be one mechanism by which MSC implantation improves post-MI cardiac function, independent of transdifferentiation to or fusion with cardiomyocytes.

Myocardial SCF production is likely important through actions on marrow-resident EPC and other precursor cells. EPCs express c-kit, whose activation by SCF is needed for EPC mobilization and function. ${ }^{19}$ Other bone marrow-derived $\mathrm{c}-\mathrm{kit}^{+}$precursor cells have also been shown to be important to neovascularization and cardiac repair. ${ }^{20,21}$ EPCs have been shown to increase in response to $\mathrm{MI}^{22,23}$ and to potentiate angiogenesis both by incorporating into new vessels and by secreting angiogenic cytokines. ${ }^{9,24,25}$ This report also demonstrates that precursor cell mobilization is greater after MSC transplantation than after coronary ligation and medium injection. Overexpression of SCF, a major antiapoptotic and survival factor, in the hostile infarct is likely to improve homing, retention, and function of the EPC and other precursor cells. ${ }^{26}$ In addition, SCF is a major

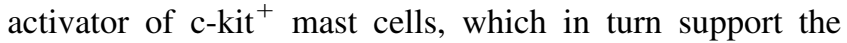
proliferation and contracture of myofibroblasts. ${ }^{27}$ Mast cells are known to infiltrate into the infarct, but their role is unclear. The SCF axis, therefore, is intimately involved in pathways that promote angiogenesis and formation of myofibroblast-rich wound repair tissue, which is critically important for progressive local remodeling and stabilization of wounds. ${ }^{28}$

The role of SCF after MI and the mechanism of benefit of MSCs overexpressing SCF were not evaluated in the current study. To address the role of SCF in the injured heart, we recently characterized the response to MI in c-kit mutant mice $\left(K i t^{W} / K_{i t}{ }^{W-v}\right)$ in which SCF binding to the c-kit receptor does not initiate intracellular signaling. We found that the myocardial scar expands rapidly in these mice, resulting in 2-fold higher mortality, overt heart failure, and marked pulmonary congestion (unpublished data). The effects were mediated by an inability of the mutant mice to 
form vessel- and myofibroblast-rich repair tissue. These findings formed the basis for the current study. Taken together, the data indicate a critical and novel role for SCF in the post-MI heart. We would speculate that SCF is required for the process of wound maturation and infarct stabilization in a dose-dependent manner.

There are limitations to the current study. Although conversion of the MSC to cardiomyocytes was not observed in our study, we did not assess the conversion of the implanted MSC to other lineages and the fate of the implanted cells remains unknown. Likewise, cell survival was not quantitatively assessed and the impact of SCF overproduction may be through increasing MSC survival. Although our in vitro phenotyping of the MSCs revealed that they did not express c-kit, it is plausible that the cells begin expressing c-kit after implantation. Similarly, the impact of SCF overproduction on other c-kit ${ }^{+}$cells was not directly evaluated in the current study. Last, the prolonged culture of the MSC before implantation may have affected the in vivo biologic impact of the cells and limited the plasticity of the MSC.

\section{Conclusion}

We believe that adult stem cell implantation did not regenerate new contractile units. Given the magnitude of the effects on ventricular function that was observed in this and all other previous studies, it is unlikely that the formation of a sufficient number of new cardiomyocytes to dramatically improve LV function could be missed. In contrast, if the mechanism of action depends on paracrine effects on angiogenesis and matrix stabilization, the magnitude of these effects could clearly be much greater than the number of surviving transplanted MSCs. Consistent with this hypothesis, the transplanted MSCs mobilized EPCs and promoted angiogenesis, which may have increased perfusion to surviving myocytes and/or enabled more rapid cardiac repair by other cells. Through these effects the MSCs modulated local remodeling, stabilized the infarct, and limited infarct expansion. The SCF pathway, by indirect effects on the formation of repair tissue through EPC and other c-kit ${ }^{+}$ cells, improved the repair process and assisted in preservation of ventricular geometry. Paracrine effects of MSCs may be one mechanism by which implantation of MSCs into a fresh infarct prevents rapid deterioration of cardiac function.

\section{References}

1. Tomita S, Li RK, Weisel RD, Mickle DA, Kim EJ, Sakai T, et al. Autologous transplantation of bone marrow cells improves damaged heart function. Circulation. 1999;100(19 Suppl):II247-56.

2. Tomita S, Mickle DA, Weisel RD, Jia ZQ, Tumiati LC, Allidina Y, et al. Improved heart function with myogenesis and angiogenesis after autologous porcine bone marrow stromal cell transplantation. J Thorac Cardiovasc Surg. 2002;123:1132-40.
3. Jiang Y, Jahagirdar BN, Reinhardt RL, Schwartz RE, Keene CD, Ortiz-Gonzalez XR, et al. Pluripotency of mesenchymal stem cells derived from adult marrow. Nature. 2002;418(6893):41-9.

4. Mangi AA, Noiseux N, Kong D, He H, Rezvani M, Ingwall JS, et al. Mesenchymal stem cells modified with Akt prevent remodeling and restore performance of infarcted hearts. Nat Med. 2003;9:1195-201.

5. Balsam LB, Wagers AJ, Christensen JL, Kofidis T, Weissman IL, Robbins RC. Haematopoietic stem cells adopt mature haematopoietic fates in ischaemic myocardium. Nature. 2004;428(6983):668-73.

6. Orlic D, Kajstura J, Chimenti S, Jakoniuk I, Anderson SM, Li B, et al. Bone marrow cells regenerate infarcted myocardium. Nature. 2001; 410(6829):701-5.

7. Smith MA, Court EL, Smith JG. Stem cell factor: laboratory and clinical aspects. Blood Rev. 2001;15:191-7.

8. Broudy VC, Lin NL, Priestley GV, Nocka K, Wolf NS. Interaction of stem cell factor and its receptor c-kit mediates lodgment and acute expansion of hematopoietic cells in the murine spleen. Blood. 1996; 88:75-81.

9. Asahara T, Murohara T, Sullivan A, Silver M, van der ZR, Li T, et al. Isolation of putative progenitor endothelial cells for angiogenesis. Science. 1997;275(5302):964-67.

10. Beltrami AP, Barlucchi L, Torella D, Baker M, Limana F, Chimenti S, et al. Adult cardiac stem cells are multipotent and support myocardial regeneration. Cell. 2003;114:763-76.

11. Orlic D, Kajstura J, Chimenti S, Limana F, Jakoniuk I, Quaini F, et al. Mobilized bone marrow cells repair the infarcted heart, improving function and survival. Proc Natl Acad Sci U S A. 2001;98:10344-9.

12. Murry CE, Soonpaa MH, Reinecke H, Nakajima H, Nakajima HO, Rubart M, et al. Haematopoietic stem cells do not transdifferentiate into cardiac myocytes in myocardial infarcts. Nature. 2004;428(6983): 664-8.

13. Kunisada T, Yoshida H, Yamazaki H, Miyamoto A, Hemmi H, Nishimura E, et al. Transgene expression of steel factor in the basal layer of epidermis promotes survival, proliferation, differentiation and migration of melanocyte precursors. Development. 1998;125:2915-23.

14. Aicher A, Heeschen C, Mildner-Rihm C, Urbich C, Ihling C, TechnauIhling $\mathrm{K}$, et al. Essential role of endothelial nitric oxide synthase for mobilization of stem and progenitor cells. Nat Med. 2003;9:1370-6.

15. Pittenger MF, Martin BJ. Mesenchymal stem cells and their potential as cardiac therapeutics. Circ Res. 2004;95:9-20.

16. Matsuura K, Wada H, Nagai T, Iijima Y, Minamino T, Sano M, et al. Cardiomyocytes fuse with surrounding noncardiomyocytes and reenter the cell cycle. J Cell Biol. 2004;167:351-63.

17. Shi D, Reinecke H, Murry CE, Torok-Storb B. Myogenic fusion of human bone marrow stromal cells, but not hematopoietic cells. Blood. 2004;104:290-4.

18. Majumdar MK, Thiede MA, Haynesworth SE, Bruder SP, Gerson SL. Human marrow-derived mesenchymal stem cells (MSCs) express hematopoietic cytokines and support long-term hematopoiesis when differentiated toward stromal and osteogenic lineages. J Hematother Stem Cell Res. 2000;9:841-8.

19. Heissig B, Werb Z, Rafii S, Hattori K. Role of c-kit/Kit ligand signaling in regulating vasculogenesis. Thromb Haemost. 2003;90: 570-6.

20. Li TS, Hamano K, Nishida M, Hayashi M, Ito H, Mikamo A, et al. CD117+ stem cells play a key role in therapeutic angiogenesis induced by bone marrow cell implantation. Am J Physiol Heart Circ Physiol. 2003;285:H931-7.

21. Wojakowski W, Tendera M, Michalowska A, Majka M, Kucia M, Maslankiewicz K, et al. Mobilization of CD34/CXCR4+, CD34/ CD117+, c-met + stem cells, and mononuclear cells expressing early cardiac, muscle, and endothelial markers into peripheral blood in patients with acute myocardial infarction. Circulation. 2004;110: 3213-20.

22. Leone AM, Rutella S, Bonanno G, Abbate A, Rebuzzi AG, Giovannini $\mathrm{S}$, et al. Mobilization of bone marrow-derived stem cells after myocardial infarction and left ventricular function. Eur Heart J. 2005;26: 1196-204.

23. Massa M, Rosti V, Ferrario M, Campanelli R, Ramajoli I, Rosso R, et al. Increased circulating hematopoietic and endothelial progenitor cells 
in the early phase of acute myocardial infarction. Blood. 2005; 105:199-206.

24. Asahara T, Masuda H, Takahashi T, Kalka C, Pastore C, Silver M, et al. Bone marrow origin of endothelial progenitor cells responsible for postnatal vasculogenesis in physiological and pathological neovascularization. Circ Res. 1999;85:221-8.

25. Ziegelhoeffer T, Fernandez B, Kostin S, Heil M, Voswinckel R, Helisch A, et al. Bone marrow-derived cells do not incorporate into the adult growing vasculature. Circ Res. 2004;94:230-8.

26. Heissig B, Hattori K, Dias S, Friedrich M, Ferris B, Hackett NR, et al. Recruitment of stem and progenitor cells from the bone marrow niche requires MMP-9 mediated release of kit-ligand. Cell. 2002; 109:625-37.

27. Gailit J, Marchese MJ, Kew RR, Gruber BL. The differentiation and function of myofibroblasts is regulated by mast cell mediators. J Invest Dermatol. 2001;117:1113-9.

28. Tomasek JJ, Gabbiani G, Hinz B, Chaponnier C, Brown RA. Myofibroblasts and mechano-regulation of connective tissue remodelling. Nat Rev Mol Cell Biol. 2002;3:349-63.

\section{Discussion}

Dr Georg Lutter (Kiel, Germany). Congratulations on this very interesting work. In your study you scrutinized angiogenesis and differentiated between the 2 study groups. Did you also analyze and count the amount of arteriogenesis, and look for special histologic signs of arteriogenesis, because you demonstrated your results about the myofibril generation?

Dr Fazel. That is a very good question. Initially the way we looked for angiogenesis was simply by identifying CD-31 or PECAM-1-expressing structures. We weren't looking in particular at arterioles. When we did the smooth muscle actin staining at a later stage we saw that perhaps there could be a difference. We did not quantify that.

Dr Robert C. Robbins (Stanford, Calif). I was a little confused because you must have sent an abstract in that discussed the use of wild-type and transgenic mice that were c-Kit mutants, but your article wasn't the same as the abstract. So what happened with the work on the c-Kit mutant knockout?

I am not sure you wanted me to ask that. I thought it was a beautiful abstract, and one of the things I was going to ask you is where was your positive control with adding the stem cell factor? You answered my question, but what happened to the knockouts?

Dr Fazel. Actually those data are very interesting. The data that Dr Robbins is referring to are from mice that have mutation in the c-Kit receptor. When c-Kit is completely knocked out, it results in death in utero. There is a spontaneously c-kit mutant mouse that has attenuated tyrosine kinase activity after binding its ligand, stem cell factor.

The interesting thing about these mice is that after coronary ligation, and I alluded to this in my presentation, the hearts went on to rapid heart failure much more so than the c-Kit wild-type controls. The mechanism, after we looked at it in detail, was a combination of angiogenesis and an absent myofibroblast proliferation response in the c-Kit mutant. That work was the basis for the current work, which we then tried to test the hypothesis: Is there some sort of therapeutic intervention utility to that approach?

Dr David H. Harpole, Jr (Durham, NC). I think it is very elegant work and very nicely done. I like the way you stepwise worked through each of your questions with another procedure. This is very interesting because one of the issues with cardiotoxicity of imatinib mesylate (Gleevec) is in the patients who had cardiotoxic chemotherapy first. With Gleevec as a blocker of c-Kit, it kind of makes this fit together very nicely so that, to tell you the truth, it might be interesting to look at cardiac damage in your animals from, say, doxorubicin (Adriamycin) or something and see whether c-Kit has a role in remodeling in those places, and that is why giving Gleevec in those patients made them worse. That is the first thing.

Second, have you tried using something like Gleevec as a blocker for c-Kit as opposed to your knockout mice?

Dr Fazel. Thank you for that question because we have done those studies following the same logic. Gleevec is a therapeutic agent used for myoproliferative disorders. It blocks c-Kit and platelet-derived growth factor receptors.

One of the arms in the study that Dr Robbins alluded to with the mutant mice was to induce c-Kit inhibition pharmacologically, and we used Gleevec in that study. The treated mice had virtually the same phenotype as the c-kit mutant. In particular, looking at it in more detail, there was global ventricular dilation after coronary ligation if the animals were treated with Gleevec. When we looked at, for example, vascular endothelial growth factor expression, we saw that after infarction vascular endothelial growth factor would go up in the wild type, but that it stayed low in the c-Kit mutant and Gleevec-treated group. In addition, we looked at total cell proliferation in the infarct and found that in wild type, again, it goes up after infarction, in c-Kit mutant it is blunted, and with Gleevec it is blunted. We know that most proliferating cells in an infarct are going to be myofibroblasts. So it fit in very nicely with those data.

Dr Yoshifumi Naka (New York, NY). I congratulate you for the impressive work in which you seem to be suggesting that angiogenesis is also an important factor. Have you ever looked into hemopoietic stem cell implantation rather than the mesenchymal stem cell?

Dr Fazel. We have not done those studies. Dr Robbins' group did part of those studies in which the question again was a similar one: Are these hemopoietic stem cells turning into cardiomyocytes? In a letter to Nature, along with Dr Murray and Dr Field, they showed that the hemopoietic stem cells, although they may alter ventricular geometry after implantation, did not in a large scale turn into heart muscle cells. So, again, it goes along with the same hypothesis. We have not tried those experiments ourselves. 
A

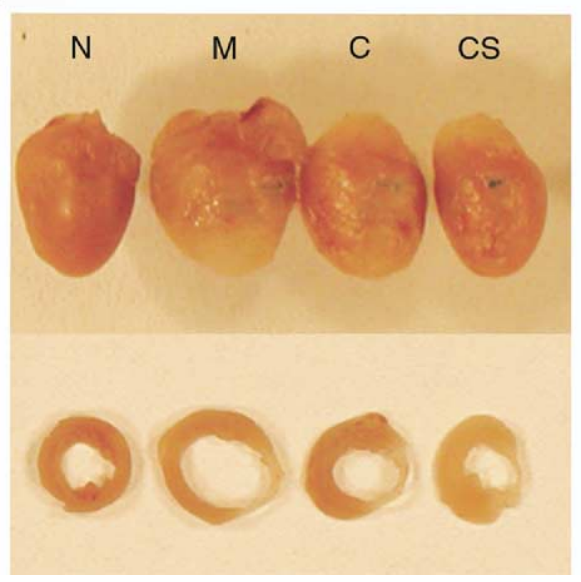

B

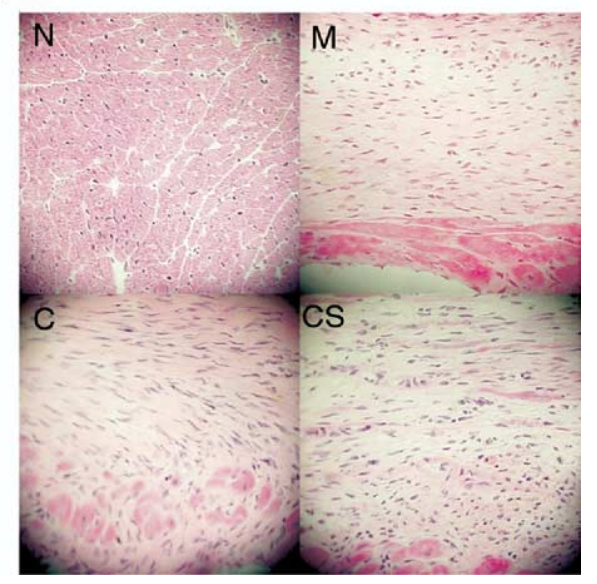

Figure E1. A, Whole heart images after in situ perfusion-fixation. The blue coronary ligature is visible and in the same place in all hearts. Transverse section taken $1 \mathrm{~mm}$ below the coronary ligature (lower pane/). Marked dilation of the medium-injected group is demonstrated. B, Histologic appearance of the scar. No obvious myocyte regeneration is apparent. Magnification: $400 \times$. N, Noninfarcted control group; $M$, medium-injected group; $C$, MSC-injected group, CS, MSC-SCF group. 